\title{
A Quasi-Monte Carlo Approach for Radial Distribution System Probabilistic Load Flow
}

\author{
Tao Cui, Franz Franchetti \\ Department of ECE, Carnegie Mellon University, Pittsburgh, PA, USA \\ Email: \{tcui,franzf $\} @$ ece.cmu.edu
}

\begin{abstract}
Monte Carlo simulation (MCS) is a numerical method to solve the probabilistic load flow (PLF) problem. Comparing to analytical methods, MCS for PLF has advantages such as flexibility, general purpose, able to deal with large nonlinearity and large variances, and embarrassingly parallelizable. However, MCS also suffers from low convergence speed and high computational burden, especially for problems with multiple random variables. In this paper, we proposed a QuasiMonte Carlo (QMC) based method to solve the PLF for radial distribution network. QMC uses samples from low-discrepancy sequence intended to cover the high dimension random sample space as uniformly as possible. The QMC method is particularly suitable for the high dimension problems with low effective dimensions, and has been successfully used to solve large scale problems in econometrics and statistical circuit design. In this paper, we showed that the PLF for radial distribution system has the similar properties and can be a good candidate for QMC method. The proposed method possesses the advantage of MCS method and significantly increases the convergence rate and overall speed. Numerical experiment results on IEEE test feeders have shown the effectiveness of the proposed method.
\end{abstract}

\section{INTRODUCTION}

With the development of smart grid technologies, the electric power distribution system plays a more active and important role in power systems. However, due to the lack of real time measurements, the integration of renewable energy resources and the emerging more active and stochastic new load such as electric vehicles, there are more and more uncertainties being introduced in distribution systems. Properly dealing with the uncertainties has become a practical challenge for distribution system planning and operation. An efficient and generally applicable computing framework that can monitor and assess the system states considering the impact of such large uncertainties would be an important tool for the reliable operation of smart distribution systems.

In order to deal with the uncertainties in the planning and operation of transmission grid, Probabilistic Load Flow (PLF) was firstly introduced in 70s [1]. The PLF models the uncertainties as input random variables (RV) with probabilistic density functions (PDF) or cumulative density functions (CDF). Based on load flow equations, it computes the output states as random variables with PDFs or CDFs [1] [2]. Recently, the PLF methods have been extensively used to analyze the distribution network operation and planning under uncertainties and to evaluate the impact of renewable energy resources and large stochastic loads. In [3], the impact of wind generation on distribution system are studied using PLF. In [4],

This work is supported by NSF through awards 0931978 and 1116802. the solar energy is modeled as a PLF problem. In [5] the electric vehicles with stochastic charging behavior are also modeled and evaluated as a PLF problem.

This paper focuses on the solution methods for PLF in distribution network. The solution methods generally fall into two categories: the analytical methods and Monte Carlo simulation (MCS) based methods [6]. Most analytical methods are trying to compute the output RVs by simplifying power system models or probabilistic models [2] [6]. Due to the simplification, analytical methods may not be able to handle uncertainties with large variance or systems with large nonlinearity. MCS is a general framework extensible for many statistical applications including solving PLF. It samples the input RVs and solves load flow for each sample using the accurate system model, and then estimates the output RVs using all result samples. The accuracy and convergence of MCS are guaranteed by the probabilistic limit theory [7]. Therefore, the MCS solutions are often used as accuracy references for most PLF researches and applications. In order to obtain converged accurate results, the MCS needs to solve a large number of load flows, due to the computational burden, MCS methods are often believed to be prohibitive for practical applications. Therefore, speeding up MCS for PLF solution and other more general applications has been an active research topic. In this paper, we proposed a Quasi Monte Carlo (QMC) based method for PLF solution of radial distribution network to improving the accuracy and overall speed of MCS based PLF solutions.

Related work. In [2] and [8], a linearization and multilinearization model is used for MCS solutions. In [9], a deterministic sampling method is used to speed up the transmission stochastic load flow. In [10], antithetic sampling is used for variance reduction in congestion forecasting. Most recently, [11] and [12] have proposed using Latin-Hypercube Sampling (LHS) method for PLF solutions for transmission and distribution network. Efforts to speedup MCS is certainly not only in PLF research field. In the domain of econometrics and statistical circuit analysis, MCS based methods are used frequently to solve the large scale problems with many random variables. To improve the efficiency, Quasi-Monte Carlo method was originally applied in financial computation, one successful QMC application is an 1439-dimensional statistical integral with a speedup of $150 \mathrm{x}$ comparing to conventional MCS methods [13]. In [14], the nano-scale statistical circuit analysis problems are effectively solved by QMC method with a $2 \mathrm{x}$ to $8 \mathrm{x}$ speedup over the conventional MCS methods.

Contribution. Inspired by the successful applications of 
QMC in different fields for similar problems. In this paper, by analyzing the property of QMC and the model of radial distribution network, we show that the radial distribution system has low effective dimension in superposition sense. The PLF for radial distribution network can be a suitable problem for QMC method to achieve better performance. We implemented and tested the QMC based PLF solver on IEEE test feeder. An up to 10x speedup on convergence speed is observed comparing to MCS method with high accuracy. Moreover, the QMC method has similar simple format as the MCS method. By switching the sample generator, QMC can be easily implemented as an alternative for MCS method.

Synopsis. The paper is organized as following: the Monte Carlo method related background are reviewed in Section II, the Quasi-Monte Carlo sampling is introduced in Section III. The model of distribution system load flow and and application of QMC solution is in Section IV. The numerical result is showed in Section V. Section VI concludes the paper.

\section{REVIEW PLF AND MCS}

\section{A. Probabilistic Load Flow}

Considering the canonical AC power flow model:

$$
\begin{aligned}
& \mathbf{0}=\mathbf{g}(\mathbf{x}, \mathbf{u}, \mathbf{A}, \mathbf{p}) \\
& \mathbf{y}=\mathbf{h}(\mathbf{x})
\end{aligned}
$$

In (1), the $\mathbf{x}$ is the system state which often contains the nodal voltages. Given the system structure $\mathbf{A}$, the parameters $\mathbf{p}$ and the generation load conditions $\mathbf{u}$, the state $\mathbf{x}$ can be exactly determined by circuit law and power conservative constraints in (1). Based on states $\mathbf{x}$, all other system states in $\mathbf{y}$ such as line flow can be exactly determined by (2). Both (1) and (2) can be nonlinear equations in $\mathrm{AC}$ power flow model.

In most planning or operation cases, due to the uncertainties and errors, not all parameters can be exactly determined for deterministic power flow analysis. Therefore Probabilistic Load Flow (PLF) models these uncertainties (usually the load generation conditions $\mathbf{u}$ ) as random variables. Based on load flow equations (1)(2), the system states and output states are also computed as random variables. The random variables are often represented by its probabilistic density function (PDF) or cumulative density function (CDF) in PLF analysis.

In general, there are two categories of solution methods for PLF problems [6]: the analytical method and Monte Carlo simulation based method. Most analytical methods are trying to compute the output RVs by simplifying power system models or probabilistic models. Such as using linearized power flow equations [1] [2], estimating certain moments or certain points of the random variables' PDF [15] [16], etc.

However, due to the simplification, analytical methods may not be able to handle uncertainties with large variance or systems with large non-linearity. In the focused area of this paper: the distribution system may have even larger nonlinearity, especially when considering the discrete control actions such as regulator tap-changing and various types of nonlinear loads. Also due to the low system capacity and small population number, the uncertainties in distribution network tend to have larger variances with more complicated distributions.

Comparing to analytical methods, the Monte Carlo simulation is a general applicable numerical solution for PLF problems. It usually consists of three steps: 1) sample the input random variables; 2) for each sample, run a deterministic load flow to obtain an accurate result sample; 3) based on all result samples, estimate the PDFs of the interested states.

The advantages of MCS method include: 1) Each sample is accurate without any approximation; 2) A general framework extensible for other statistical or non-statistical applications; 3) Has straightforward formulation and natural interpretations. Therefore, MCS is often used as accuracy references for most PLF research and applications [2] [15] [16].

The major drawback of MCS method is the computational burden, which almost restrict MCS method for only off-line comparison study. However, recent study in Monte Carlo simulation has show that certain sampling method such as Quasi-Monte method can be an efficient alternative for certain problems. Moreover, MCS method can also be classified as the embarrassingly parallelizable problem for modern parallel computing hardware [17] [18], resulting very efficient solver from hardware perspective. Therefore, the MCS method for PLF solution and its properties are worth to be revisited.

\section{B. Estimating Density Functions in Probabilistic Load Flow}

In order to estimate the CDF of certain state variable $X$, consider the indicator function $\mathbf{1}_{a}(X): X \rightarrow\{0,1\}$ such that:

$$
\mathbf{1}_{a}(X)= \begin{cases}1 & \text { if } X \leq a, \\ 0 & \text { if } X>a .\end{cases}
$$

Since the CDF of $X$ at value $a$ is defined as

$$
F_{X}(a)=\mathrm{P}(X<a)=\mathrm{E}\left[\mathbf{1}_{a}(X)\right]=\int_{X} \mathbf{1}_{a}(x) d \mathbb{P}
$$

If above $x$ is random sample draw from the distribution of random variable $X$. Then (3) can be regarded as a CDF estimator with unbiased mean $\mathrm{E}\left(\mathbf{1}_{a}(X)\right)=P(X<a)$ and limited variance:

$$
\begin{aligned}
\operatorname{Var}\left(\mathbf{1}_{a}(X)\right) & =E\left(\left(\mathbf{1}_{a}(X)-P(X<a)\right)^{2}\right) \\
& =\mathrm{P}(X<a) \cdot(1-\mathrm{P}(X<a))
\end{aligned}
$$

From numerical simulation point of view, estimating $F_{X}(a)$ is the same as evaluating the expectation (or calculating the integral part) in (4). The MCS method is a standard way for such expectation estimation (or integral) problem. For continuous variables, PDFs are the derivatives of CDFs.

\section{Convergence of MCS}

To evaluate a general high dimension integral problem:

$$
I=\int_{R^{d}} f(x) d x \quad R^{d}=[0,1]^{s}
$$

MCS compute the approximated:

$$
I_{n}=\frac{1}{n} \sum_{i=1}^{n} f\left(x_{i}\right) \quad x_{i} \in R^{d}
$$


$x_{1}, \ldots, x_{n}$ are independent and identically distributed samples randomly draw from the $d$ dimension space $R^{d}$. By central limit theorem:

$$
\lim _{n \rightarrow \infty} I_{n}=I
$$

and the mean square error is:

$$
\begin{aligned}
M S E_{n} & =\frac{1}{n^{2}} \sum_{i=1}^{n} E\left[\left(f\left(x_{i}\right)-E\left(f\left(x_{i}\right)\right)\right)^{2}\right] \\
& =\operatorname{Var}\left(f\left(x_{i}\right)\right) / n=\sigma_{f}^{2} / n
\end{aligned}
$$

We know the variance $\sigma_{f}^{2}$ for CDF is finite in (5). From central limit theory, the standard deviation of MCS estimator is $\sigma_{f} / \sqrt{n}$. Therefore, the error in MCS decreases asymptotically to 0 at the rate $n^{-1 / 2}$. This also means the increase of accuracy may require much more increase of the samples.

Variances reduction techniques such as important sampling, stratified sampling, antithetic sampling, etc are typically aiming to reduce the $\sigma_{f}$ part of the MCS error. While for estimation of the full density function, it is usually difficult to find such a variance reduction technique for all density points.

Moreover, the error of MCS is given in the sense of probability, which means on average MCS may perform well with expected accuracy, but there is possibility that particular sequence of random samples may lead to unacceptable errors.

Comparing to conventional MCS, Quasi-Monte Carlo method turns to be a better choice for particular PLF problem for radial distribution network because:

1) QMC reduces the error by speedup the $n^{-1 / 2}$ part, which accommodates all points of the density function.

2) QMC is based on deterministic samples, the error is bounded by asymptotically decreasing upper bound.

3) The radial structure distribution network can have lower effective dimensions which is necessary for QMC to achieve better results.

We will explain the above in detail in following sections.

\section{QUasi-Monte CARLo (QMC) AND KERnEL DENSITY ESTIMATION (KDE)}

The main difference of QMC and MCS method is the sampling method. MCS uses the sample numbers generated from pseudo-random number generators (PRNG), while QMC uses sample numbers from low-discrepancy sequence (LDS), also called quasi-random number generators (QRNG).

\section{A. Low-Discrepancy Sequence}

Fig. 1 shows the uniform distribution random samples in 50 dimension hypercube $[0,1)^{50}$. On the left is the first two dimension point $\left(x_{1}, x_{2}\right)$, on the right is the histogram of first dimension $x_{1}$. Fig. 1(a) shows the samples generated by standard Mersenne Twister (MT) PRNG. Fig. 1(b) shows the samples generated by Sobol sequence QRNG.

Clearly, the random samples by MT PRNG do show "randomness", but the points are not equally distributed over the space. The histogram is not even close to the uniform distribution based on these 500 samples. The samples by Sobol QRNG are not random, but are equally distributed over the space, and the histogram shows a close-to uniform distribution.
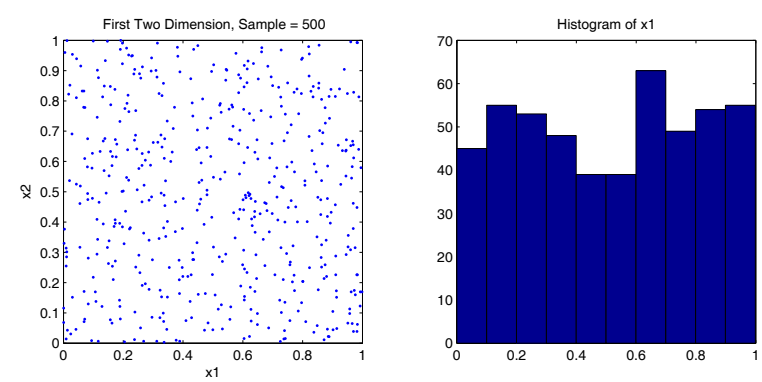

(a) Pseudo-random number generation for MCS
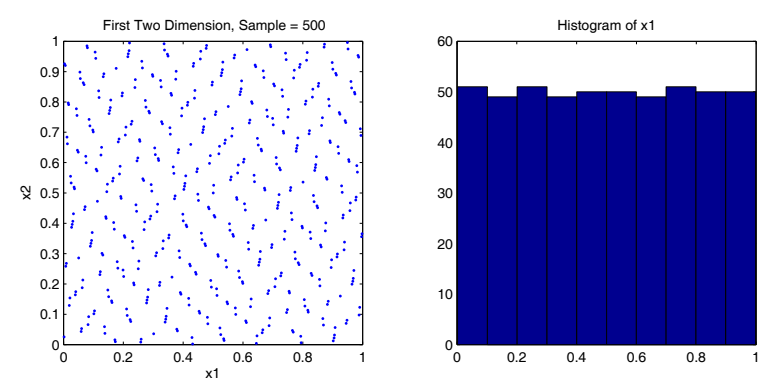

(b) Quasi-random number generation for QMC

Fig. 1. Comparison of PRNG and QRNG samples

\section{B. Error of Integral Approximation using Samples}

Consider the general problem: approximation of integral by sampling method. The error is bounded by a term proportional to the discrepancy of the sample set $\left(x_{1}, \ldots, x_{N}\right)$ and total variation of the function. The Koksma-Hlawka inequality shows the error $\epsilon$ of integral function $f(x)$ over hypercube $[0,1)^{s}$ :

$$
\epsilon=\left|\int_{[0,1)^{s}} f(u) \mathrm{d} u-\frac{1}{N} \sum_{i=1}^{N} f\left(x_{i}\right)\right|
$$

is bounded by

$$
|\epsilon| \leq V(f) D_{N}
$$

Where $V(f)$ is the Hardy-Krause variation related to the total variation of $f$ over the high dimension hypercube. $D_{N}$ is the discrepancy of the sample set $\left(x_{1}, \ldots, x_{N}\right)$ and can be defined as (so-called Star Discrepancy [19]):

$$
D_{N}=\sup _{Q \subset[0,1)^{s}}\left|\frac{\text { number of points in } Q}{N}-\operatorname{volume}(Q)\right|
$$

The Koksma-Hlawka inequality separates the estimation error into two parts: the proportional part $V(f)$ depending on the integral function, and the discrepancy part $D_{N}$ depending on the sample set. And it applies to the general problem and method for integral approximation including MCS, QMC as well as standard numerical integration methods [19].

\section{Kernel Density Estimation}

Kernel density estimation (KDE) is a generally applicable non-parametric way of estimating the probability density function of a random variable [20]. The general formulation is:

$$
\hat{f}_{h}(x)=\frac{1}{n h} \sum_{i=1}^{n} K\left(\frac{x-x_{i}}{h}\right)
$$


Where $K$ is the kernel function, $x_{i}$ is the value of sample point $i, h$ is the bandwidth. $\hat{f}_{h}(x)$ is the estimated density function. We choose Gaussian function as the kernel and use the optimized bandwidth for Gaussian distribution [20].

The assumption for using KDE is that the resulting density function is smooth enough. Using KDE in density estimation is equivalent to replacing the indicator function with a smooth differentiable kernel function. Once apply KDE with preset bandwidth in density estimation, the function variation $V(f)$ can become finite. Therefore, from (12), QMC may have asymptotically faster convergence rate comparing to MCS.

\section{Theoretical Bound}

In our proposed approach, we use low-discrepancy sequence of QMC for the quasi random number generation and KDE for the density estimation. QMC tries to reduce $D_{N}$ part of the error to increase the convergence speed. The best-known LDS can have the discrepancy bounded by $O\left(\frac{\log (N)^{s}}{N}\right)$. Therefore, the QMC result has an error bounded $O\left(\frac{\log (N)^{s}}{N}\right)$, where $s$ is the problem dimension and $N$ is the number of samples.

While it has been showed that the pseudo-random number sequence used by Monte Carlo simulation has the discrepancy bounded by $O\left(\left[\frac{\log \log N}{N}\right]^{1 / 2}\right)$ with probability 1 [21]. This also matches the Monte Carlo MSE $O\left(\frac{1}{\sqrt{N}}\right)$ in (9).

Therefore, one can say that for a fixed dimension $s$, QMC converge with error bound at $O\left(\frac{\log (N)^{s}}{N}\right)$, which is faster than MCS' error bound $O\left(\left[\frac{\log \log N}{N}\right]^{1 / 2}\right)$ and MSE $O\left(\frac{1}{\sqrt{N}}\right)$.

\section{E. Simple QMC-KDE Example for PDF Estimation}

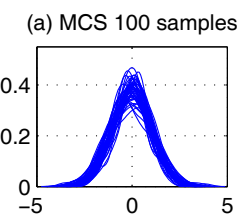

(d) QMC 100 samples

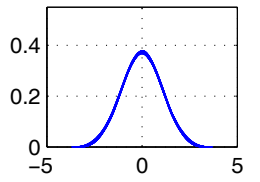

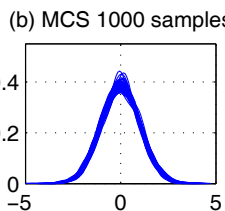

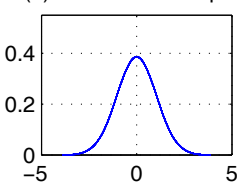

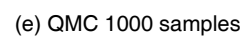

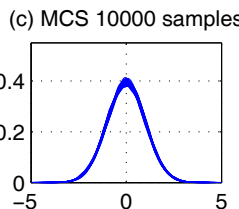

(f) True PDF

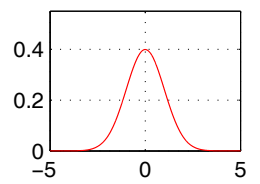

Fig. 2. Example of MCS and QMC to estimate Normal distribution

Fig. 2 shows an example of comparing MCS and QMC using KDE to estimate PDF. We generate samples from Normal distribution: $N(0,1)$, and use these samples to estimate the original PDF curve. From (a) to (e), for each plot, we generate 100 different sequences of samples. For each sequence of samples, a PDF curve is estimated and plotted, each plot has 100 PDF curves. In (a) to (c), the samples are generated from MT PRNG. In (d) and (e), the samples are generated from Sobol QRNG. Clearly we can see the PDF estimated from QMC converge to the single true PDF with much less samples.

The promising result motivates many applications of QMC method. However, in order to apply QMC on practical problems for better performance, special problem formulation is necessary. For example, a bigger dimension $s$ may require much bigger $N$ for QMC to outperform MCS. Fortunately in practice, many high dimension problems can have lower effective dimension, and can be suitable for QMC method. We will show in the following section that the probabilistic load flow problem in radial distribution network can be a good candidate for QMC to achieve good performance.

\section{LEVERAGING QMC FOR RADIAL DistribUtion NETWORK PLF SOLUTIONS}

\section{A. Effective Dimensions for $Q M C$}

The effective dimension has been used to explain the success of QMC on many practical problems including statistical circuit analysis and many other general problems [14] [19].

The effective dimension is highly related to analysis of variance (ANOVA). In ANOVA, the multivariate function can be decomposed into multiple functions of lower dimensions.

$$
f(\mathbf{u})=\sum_{I \subseteq\{1, \ldots, s\}} f_{I}(\mathbf{u})
$$

The variance of ANOVA component is:

$$
\sigma_{I}^{2}=\int_{[0,1)^{s-d}} f_{I}(u)^{2} \mathrm{~d} u_{I}
$$

All the components are orthogonal, and the total variance of $f$ can be written as:

$$
\operatorname{Var}(f)=\sigma^{2}=\sum_{I} \sigma_{I}^{2}
$$

The ANOVA decomposes the variance of high dimension function $f$ into sum of variances of lower dimension components. From (16), the effective dimension is defined as:

The effective dimension in superposition sense is the smallest integer $d_{S}$ such that:

$$
\sum_{0<|u| \leq d_{S}} \sigma_{u}^{2}=p \sigma^{2}
$$

The effective dimension in truncation sense is the smallest integer $d_{T}$ such that:

$$
\sum_{u \subseteq\left\{1, \ldots, d_{T}\right\}} \sigma_{u}^{2}=p \sigma^{2}
$$

Where $p$ is a number close to 1 (e.g. $p=0.99$ ).

Intuitively, the effective dimension means that the function with an effective dimension of $d$ can be well approximated by sum of functions of at most $d$ variables (in superposition sense), or sum of functions involving only the first $d$ variables $u_{1}, \ldots, u_{d}$ (in truncation sense) [19]. For example: let $\mathbf{x}=$ $\left(x_{1}, x_{2}, x_{3}\right)$, the 3 dimension function.

$$
f(\mathbf{x})=f_{1}\left(x_{1}\right)+f_{2}\left(x_{2}\right)+f_{3}\left(x_{4}\right)
$$

has the effective dimension of 1 in superposition sense, since $f(\mathbf{x})$ is the sum of functions with at most 1 variable. 


\section{B. Radial Distribution Network Model}

We have following assumptions for distribution system PLF.

1 A radial tree structure network, the substation at the root is the slack bus where the voltages are assumed given.

2 The randomness is from power injections or current injections (stochastic load or renewable generations).

3 Comparing to the power injection, the power loss on the network is very low.

Above assumptions are hold for many load flow and probabilistic load flow analysis in distribution network [22]. The general formula of distribution network probabilistic load flow can be written as following:

$$
\left(V_{1}, V_{2}, \ldots, V_{N}\right)=f\left(P_{1}^{s}, P_{2}^{s}, \ldots, P_{m}^{s}, P_{1}^{r}, P_{2}^{r}, \ldots, P_{n}^{r}\right)
$$

Where $V_{i}$ is the state to be solved. $P_{i}^{s}$ is the specified parameter such as fixed power injection. $P_{i}^{r}$ is the random parameter such as random power injection described by a PDF. To solve the PLF using Monte Carlo simulation method is to generate samples of these random variables and evaluate integration. Therefore, the dimension is the total number of random variables: $n$. However, effective dimension can be much smaller than $n$, as shown in following paragraphs:

The tree structure with slack bus as the root means for any particular bus in the network, there is only one path from the root to that bus. To solve the state on that bus, the full network can be reduced to a compact network which only includes the path from root. Other branches can be aggregated as a nodal power injection at the branching points.

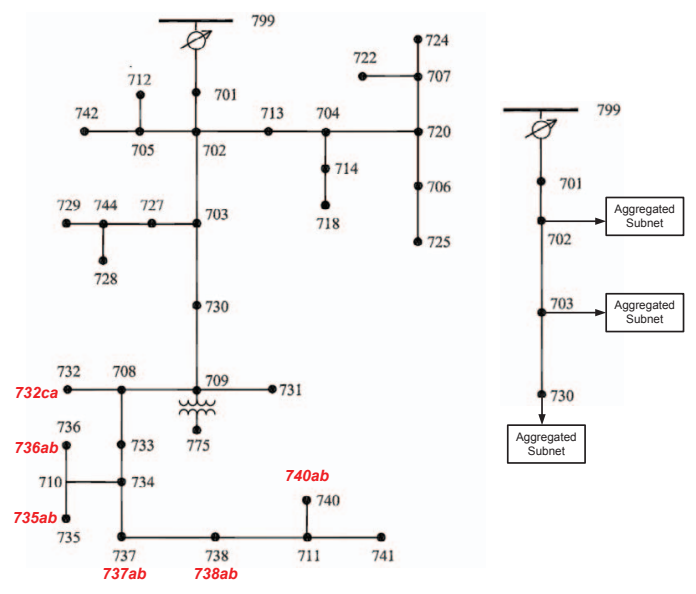

Fig. 3. IEEE 37-bus Test Feeder (Left: Full, Right: Reduced)

For example, suppose we are interested in solve the states of Bus 730 in Fig. 3, all the downstream buses from 730 can be aggregated into one nodal power injection at Bus 730. Also, all other branches on Bus 702 and 703 can be aggregated into nodal power injections on Bus 702 and Bus 703. Therefore, in the right figure in Fig. 3, the network for solving the state on Bus 730 has been reduced to a 5-bus system.

The aggregated nodal power injection are the total power injections in the reduced subnet plus the losses on the subnet. An equation similar to (19) can be written to represents such relations: $P_{\text {subnet }}=f_{1}\left(P_{s 1}\right)+\ldots f_{N}\left(P_{s N}\right)+P_{\text {loss }}$. Where $P_{s i}$ is the power injection on node i in the subnet. As we already assume that the losses on the subnet is small comparing to the power injection. By ignoring the loss' contribution to the variance of the state, $P_{\text {subnet }} \simeq f_{1}\left(P_{1 s}\right)+\ldots f_{N}\left(P_{N s}\right)$. Similar to (19), the effective dimension in superposition sense can be actually reduced to the dimension of $P_{s i}$. The possible value of the effective dimension of the subnet is 3 if we consider there are random active power injections on all three phases.

Moreover, in PLF analysis, usually not all power injections are random variables, therefore, the effective dimensions of PLF on distribution networks are usually much less than the actual number of random variables. As a result, the QMC can be expected to have a better performance on PLF for radial distribution network than conventional MCS.

\section{NUMERICAL EXPERIMENTS}

\section{A. Simulation Setup}

We use IEEE 37-bus test feeder to test our proposed method. Detail model of the network can be found in [23] [24]. In our simulation, the system configuration is the same as in OpenDSS software [24], except all the loads are modeled as PQ load. The multiple random active power injections are attached to the following locations: 732 Phase CA, 735 Phase AB, 736 Phase AB, 737 Phase AB, 738 Phase AB and 740 Phase AB. All the random loads are modeled as Normal distribution with zero mean and $50 \mathrm{~kW}$ standard deviation.

In our test, given each sample number limit, we generate 10 different solutions using different sample sequence. According to probabilistic limit theories, these 10 PDF results converge to the true PDF with large enough sample number limit.

\section{B. Accuracy and Convergence Speed}

In order to show the accuracy and convergence of MCS and QMC for the above case setup, we pick two typical buses in the network: the 703 closer to the root and 738 in the center of the random loads. We compare the PDF results of phase A's voltages of both buses in Fig. 4 and Fig. 5. Other buses' PDF results are very similar to these two figures. In these figures, with increasing sample numbers, all ten PDF curves converges to one single curves for both MCS and QMC. However, the QMC results require much less samples to achieve same accuracy comparing to the MCS method.

We further calculate the normalized error band of all voltage PDFs in the system and shown in Fig. 6. The normalized error band is the maximal difference of the all 10 PDFs' density value divided by the maximal value of the PDF curves for each case. It shows the maximal variation of the result PDF curves using different sample sequence. For converged PDFs, this value should be closer to zero.

In Fig. 6, to achieve the same level of accuracy, MCS requires 10x more samples comparing to $\mathrm{QMC}$, which means much more load flow computation than QMC. QMC for this case can achieve 10x speedup comparing to MCS method. 

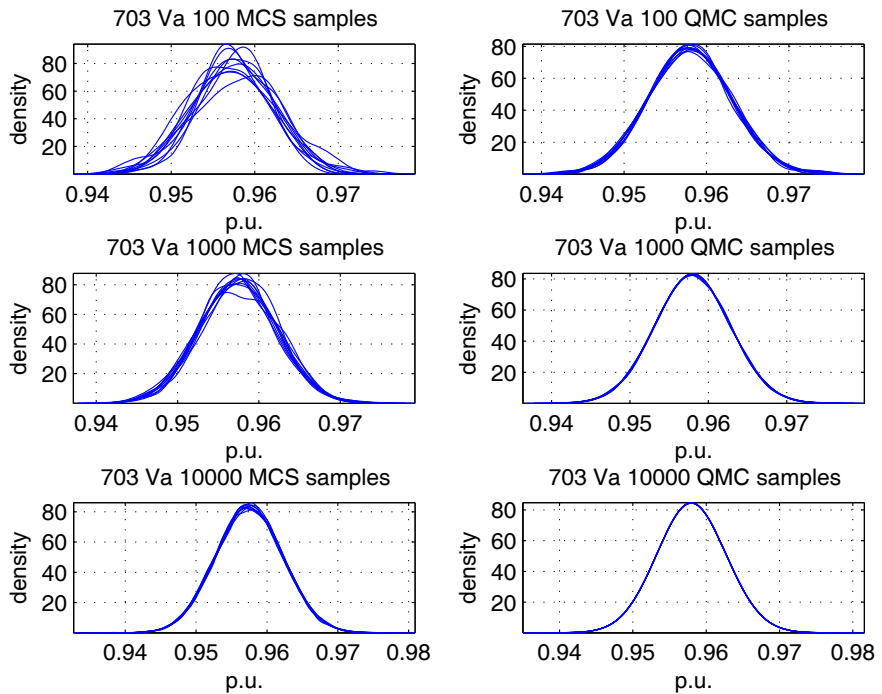

Fig. 4. Comparison of MCS and QMC (Bus 703, Phase A)
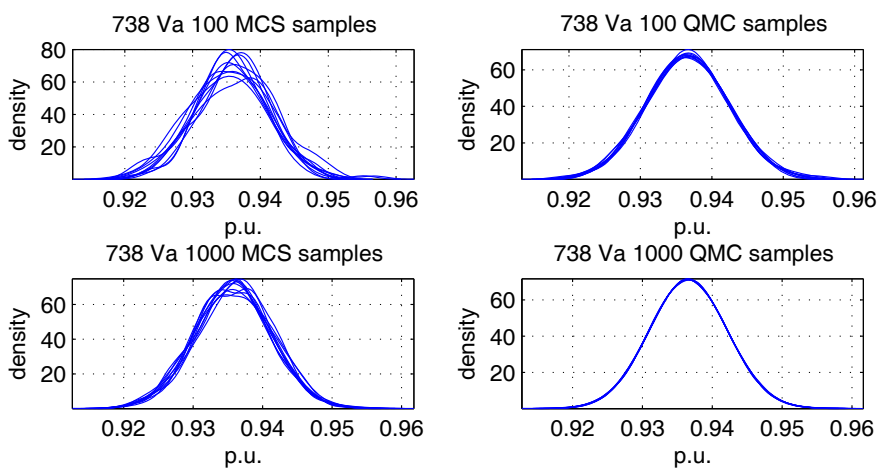

738 Va 10000 MCS samples
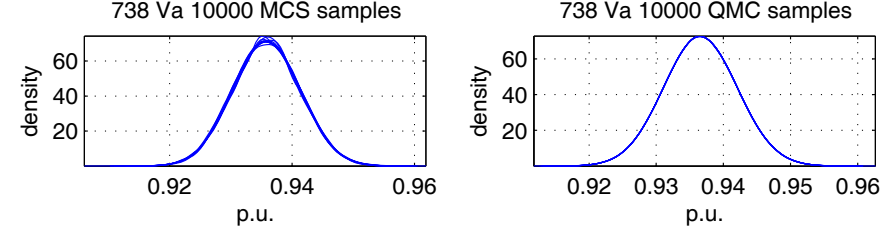

Fig. 5. Comparison of MCS and QMC (Bus 738, Phase A)

\section{CONCLUSION}

In this paper, we proposed a Quasi-Monte Carlo method to solve the PLF for radial distribution networks. QMC uses samples from low-discrepancy sequence to cover the high dimension random sample space as uniform as possible. We showed that the PLF for radial distribution system is suitable for QMC method. The proposed method possesses the advantages of MCS method and significantly increases the overall speed. Numerical experiment results on IEEE test feeders have
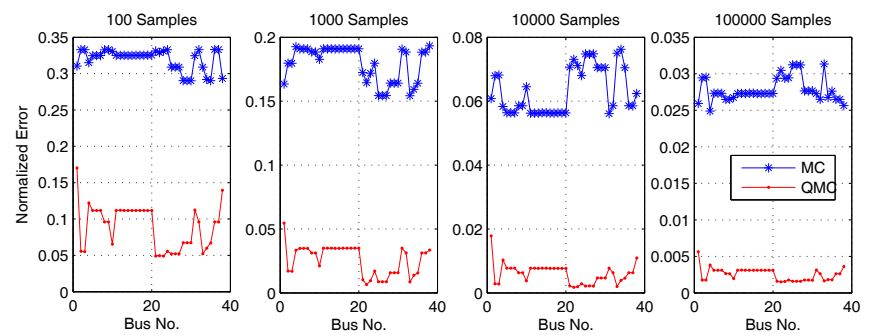

Fig. 6. Normalized error band on all buses' phase A voltage PDFs shown the effectiveness of the proposed method. Therefore, QMC can be an efficient tool for evaluating the impact of integration of stochastic renewable energy resources and loads with large variance in the smart distribution networks.

\section{REFERENCES}

[1] B. Borkowska, "Probabilistic load flow," Power Apparatus and Systems, IEEE Transactions on, vol. PAS-93, no. 3, pp. 752 -759, May 1974.

[2] R. Allan, A. Leite da Silva, and R. Burchett, "Evaluation methods and accuracy in probabilistic load flow solutions," Power Apparatus and Systems, IEEE Transactions on, vol. 100, no. 5, pp. 2539 -2546, 1981.

[3] P. Caramia, G. Carpinelli, M. Pagano, and P. Varilone, "Probabilistic three-phase load flow for unbalanced electrical distribution systems with wind farms," Renewable Power Generation, IET, vol. 1, no. 2, 2007.

[4] F. Ruiz-Rodriguez, J. Herna andndez, and F. Jurado, "Probabilistic load flow for radial distribution networks with photovoltaic generators," Renewable Power Generation, IET, vol. 6, no. 2, pp. 110 -121, 2012.

[5] G. Li and X.-P. Zhang, "Modeling of plug-in hybrid electric vehicle charging demand in probabilistic power flow calculations," Smart Grid, IEEE Transactions on, vol. 3, no. 1, pp. 492 -499, March 2012.

[6] P. Chen, Z. Chen, and B. Bak-Jensen, "Probabilistic load flow: A review," in Electric Utility Deregulation and Restructuring and Power Technologies, 2008. DRPT 2008. Third International Conference on, April 2008, pp. $1586-1591$.

[7] J. Liu, Monte Carlo strategies in scientific computing. Springer, 2008.

[8] A. Leite da Silva and V. Arienti, "Probabilistic load flow by a multilinear simulation algorithm," Generation, Transmission and Distribution, IEE Proceedings C, vol. 137, no. 4, pp. 276 -282, Jul 1990.

[9] H. Liao, "Fast deterministic sampling for mean and covariance estimation in stochastic load flow," in Power Engineering Society General Meeting, 2007. IEEE, June 2007.

[10] G. Zhang, B. Zhang, H. Sun, and W. Wu, "Ultra-short term probabilistic transmission congestion forecasting considering wind power integration," in Advances in Power System Control, Operation and Management (APSCOM 2009), 8th International Conference on, nov. 2009.

[11] H. Yu, C. Chung, K. Wong, H. Lee, and J. Zhang, "Probabilistic load flow evaluation with hybrid latin hypercube sampling and cholesky decomposition," Power Systems, IEEE Transactions on, vol. 24, no. 2, pp. $661-667,2009$.

[12] J. Cao, W. Du, H. Wang, and L. Xiao, "Probabilistic load flow using latin hypercube sampling with dependence for distribution networks," in Innovative Smart Grid Technologies (ISGT Europe), 2011 2nd IEEE PES International Conference and Exhibition on, Dec. 2011.

[13] S. Ninomiya and S. Tezuka, "Toward real-time pricing of complex financial derivatives," Applied Mathematical Finance, vol. 3, no. 1, 1996.

[14] A. Singhee and R. Rutenbar, "Why quasi-monte carlo is better than monte carlo or latin hypercube sampling for statistical circuit analysis," Computer-Aided Design of Integrated Circuits and Systems, IEEE Transactions on, vol. 29, no. 11, pp. 1763 -1776, 2010.

[15] P. Zhang and S. Lee, "Probabilistic load flow computation using the method of combined cumulants and gram-charlier expansion," Power Systems, IEEE Transactions on, vol. 19, no. 1, pp. 676 - 682, 2004.

[16] C.-L. Su, "Probabilistic load-flow computation using point estimate method," Power Systems, IEEE Transactions on, vol. 20, no. 4, pp. 1843 - 1851, 2005.

[17] T. Cui and F. Franchetti, "A multi-core high performance computing framework for distribution power flow," in North American Power Symposium (NAPS), 2011. IEEE, 2011.

[18] $\_$,A multi-core high performance computing framework for probabilistic solutions of distribution systems," in Power and Energy Society General Meeting, 2012 IEEE, 2012.

[19] C. Lemieux, Monte Carlo and Quasi-Monte Carlo Sampling. Springer, 2009.

[20] B. Silverman, Density estimation for statistics and data analysis. Chapman \& Hall/CRC, 1986, vol. 26

[21] J. Halton, "On the efficiency of certain quasi-random sequences of points in evaluating multi-dimensional integrals," Numerische Mathematik, vol. 2, no. 1, pp. 84-90, 1960.

[22] W. Kersting, Distribution system modeling and analysis. CRC, 2006.

[23] IEEE PES Distribution System Analysis Subcommittee, "Distribution test feeders," http://ewh.ieee.org/soc/pes/dsacom/testfeeders/index.html.

[24] EPRI "OpenDSS" http://sourceforge.net/projects/electricdss/. 